\title{
Immunohistochemical Characterization of Normal Ovary and Common Epithelial Ovarian Neoplasm with a Monoclonal Antibody to Cytokeratin and Vimentin
}

\author{
Ankita Goel ${ }^{1 *}$, Nandam Mohan Rao ${ }^{1}$, Vissa Santhi ${ }^{1}$, Syam Sundar byna ${ }^{1}$, Bhavana Grandhi ${ }^{1}$, \\ Jyothi conjeevaram ${ }^{1}$ \\ 1. Dept. of preventive and social medicine,Narayana medical college and hospital, Nellore, India
}

\begin{tabular}{c} 
KEYWORDS \\
\hline Cytokeratin \\
Vimentin \\
Epithelial Tumor \\
Ovary \\
Benign \\
Borderline \\
Malignant \\
\\
\\
Article Info \\
\hline
\end{tabular}

Received 06 Dec 2016;

Accepted 26 Apr 2017;

Published Online 2017;

\author{
ABSTRACT
}

Background \& Objective: The common epithelial ovarian tumors are classified into serous, mucinous, clear cell, endometrioid, the Brenner, mixed, and undifferentiated types. Cytoskeleton intermediate filament composition of ovarian tissues indicates that the cytokeratin and vimentin are observed in ovarian surface epithelium along with the common ovarian epithelial tumors. The current study aimed at investigating the cytokeratin and vimentin expression in epithelial ovarian tumors to establish a diagnostic relevance.

Methods: Sixty-six common epithelial ovarian tumors were studied using anticytokeratins (Monoclonal Mouse Anti-Human Cytokeratin Clones AE1/AE3; DAKO, Denmark,) and anti-vimentin (Monoclonal Mouse Anti-Vimentin, Clone V9; DAKO, Denmark,) to ascertain the intermediate filament profiles in formalinfixed and paraffin-embedded surgical pathology materials.

Results: All ovarian epithelial tumors expressed cytokeratin in a uniform fashion. Vimentin was coexpressed with high intensity in $62.5 \%$ of serous carcinomas, mild intensity in $25 \%$ of mucinous adenocarcinoma, and moderate intensity in single case of endometrioid adenocarcinoma. Vimentin decoration in mucinous carcinoma had a focal involvement, whereas malignant endometrioid and serous decoration tended to involve larger areas. There was a significantly increased expression of vimentin in serous cystadenoma and serous carcinoma, compared with their mucinous counterparts. Also, vimentin expression and histologic grade of serous tumors showed a positive correlation. No association was found between vimentin expression and degree of differentiation in mucinous, endometrioid, and Brenner tumors.

Conclusion: The current investigation emphasized the efficiency of immunohistochemistry (IHC) typing as a tool for a more precise characterization of the origin and differentiation of human neoplasms.

Corresponding Information: Dr. Ankita Goel, Assistant professor, Department of Pathology, Narayana-Medical College and Hospital, Nellore (524002), Email: ankigoel88@gmail.com, Tel: 09988218311

Copyright $\odot$ 2017, IRANIAN JOURNAL OF PATHOLOGY. This is an open-access article distributed under the terms of the Creative Commons Attribution-noncommercial 4.0 International License which permits copy and redistribute the material just in noncommercial usages, provided the original work is properly cited.

\section{Introduction}

The epithelial tumors of the ovary are derived from surface epithelium of the ovary. They are classified into serous, mucinous, clear cell, endometrioid, the Brenner, mixed, and undifferentiated types and are graded into benign, borderline, and malignant categories (1). Cytokeratin (CK) and vimentin are the main intermediate filaments or cytoskeleton proteins found in mammalian cells. Cytokeratin is found in the epithelial cells and their tumors. Cytokeratin consists of a family of 20 different polypeptides, numbered 1 to 20 , according to differences in molecular weight and isoelectric $\mathrm{pH}$. Vimentin is a $57-\mathrm{kD}$ intermediate filament, which possesses specificity for the cells of mesenchymal origin. The 
coexpression of intermediate filaments particularly cytokeratin and vimentin isobserved in numerous normal and neoplastic tissue (2-4). Thus, the current study aimed at investigating 66 common epithelial ovarian tumors to ascertain the frequency and patterns of intermediate filament coexpression (cytokeratin and vimentin) and characterizing these tumors to determine the diagnostic relevance, particularly in distinguishing benign, borderline, and malignant epithelial tumors of ovary.

\section{Materials and methods}

Sixty-six common epithelial tumors of the ovary were obtained from the gyn-pathology records of Narayana Medical College and Hospital, Nellore, India, from January 2013 to January 2015. At least twoparaffin blocks were available from each case. One normal ovary fixed in buffered formalin was studied. The epithelial tumors of ovary were classified and graded according to histologic classification of ovarian tumors by the World Health Organization (WHO) into benign, borderline, and malignant grades of serous, mucinous, endometrioid, clear cell, and transitional cell tumors, as well as carcinosarcoma in epithelial stromal tumors (4).

Normal tissue and neoplasms were studied using anti-cytokeratins AEI/AE3 (Monoclonal Mouse Anti-Human Cytokeratin Clones AE1/AE3; DAKO, Denmark), and anti-vimentin (Monoclonal Mouse Anti-Vimentin, Clone V9; DAKO, Denmark).

Four micron paraffin-embedded tissue sections of normal and neoplastic were applied to glass slides (Biogenex optiplus ${ }^{\mathrm{TM}}$ microscope slides) coated with $0.1 \%$ poly-D-lysine. The tissue sections were immunohistochemically stained using an avidinbiotin peroxidase complex (ABC) method. AEI/AE3 (pancytokeratin) and vimentin antibody were utilized at a dilution of 1:50 in buffer.

The sections were rehydrated by sequential immersion in xylene, graded concentrations of ethanol, and tap water. Slides were kept in citrate buffer ( $\mathrm{pH}$ 9) and two cycles of heat retrieval were done in oven at $99^{\circ} \mathrm{C}$ for ten and five minutes, respectively. Slides were washed in Tris buffer $(\mathrm{pH}$ 7.8). All tissue sections were incubated with hydrogen peroxide for 10 minutes to eliminate endogenous peroxidase activity. Sections were washed thrice in Tris buffer, followed by 30 minutes incubation with cytokeratin and vimentin antibodies. Secondary antibody (Dako REAL ${ }^{\mathrm{TM}}$ Envision $^{\mathrm{TM}}$ ) was added after washings with Tris buffer for 40 minutes. At the end, chromogen diaminobenzidine (DAB) was added for 10minutes, followed by counterstaining with hematoxylin for two minutes, sequential immersions in xylene and alcohol and mounting with distyrene plastisizer xylene (DPX).

To rule out instability of reagents, positive and negative controls were run simultaneously with patient's specimen. If unexpected staining was observed, which cannot be explained by variations in laboratory procedures and a problem with the antibody was suspected, the test was discarded and a new test was performed again with a new kit.

Normal cervical squamous epithelium served as a positive control for cytokeratin and myometrium as a positive control for vimentin. The used negative control was Dako Mouse IgG1, code number X 0931, diluted to the same mouse $\mathrm{IgG}$ concentration as the primary antibody.

Hispathological examination and immunehistochemical characterization were done by two pathologists, individually, to reduce observer bias.

Based on the intensity of color produced by staining in more than $50 \%$ of cell population, it was scored as follows:

$1+$, weak staining

$2+$, moderate staining

$3+$, strong staining

Data were collected and evaluated using the statistical package SPSS version 22 for Windows (SPSS Inc., Chicago, IL). The Fisher exact test was used to analyze the data and P-value was calculated wherever required. $P$-value $\leq 0.05$ was considered statistically significant.

\section{Results}

According to histologic classification of ovarian tumors by WHO, ovarian epithelial tumors were classified into 41 serous tumors ( 28 benign, five borderline, and eight malignant), 17 mucinous tumors (seven benign, six borderline, and four malignant), six endometrioid tumors (five benign 
and one malignant) and two transitional cell tumors with one benign and one borderline grade. No clear cell tumor and epithelial stromal tumor were detected (Table1)

Table 1. Detection of Cytokeratin and Vimentin in Common Epithelial Ovarian Neoplasms

\begin{tabular}{|c|c|c|c|c|c|c|c|c|c|}
\hline \multirow[t]{2}{*}{ Tumor } & \multirow[t]{2}{*}{ Number } & \multicolumn{4}{|c|}{ Cytokeratin Grade } & \multicolumn{3}{|c|}{ Vimentin Grade } & \multirow[t]{2}{*}{ P-value } \\
\hline & & $3+$ & $2+$ & $1+$ & $3+$ & $2+$ & $1+$ & Negative & \\
\hline Serous tumors & 41 & & & & & & & & \\
\hline Benign (cystadenoma) & 28 & 28 & 0 & 0 & 0 & 0 & 4 & 24 & \multirow{3}{*}{$<0.001$} \\
\hline Serous borderline tumor & 5 & 5 & 0 & 0 & 0 & 3 & 2 & 0 & \\
\hline Serous adenocarcinoma & 8 & 4 & 1 & 3 & 5 & 2 & 1 & 0 & \\
\hline Mucinous tumors & 17 & & & & & & & & \\
\hline Benign(cystadenoma) & 7 & 7 & 0 & 0 & 0 & 0 & 1 & 6 & \multirow{3}{*}{$<0.001$} \\
\hline Mucinous borderline tumor & 6 & 5 & 1 & 0 & 0 & 0 & 4 & 2 & \\
\hline Mucinous adenocarcinoma & 4 & 3 & 0 & 1 & 0 & 0 & 1 & 3 & \\
\hline Transitional cell tumors & 2 & & & & & & & & \\
\hline Brenner tumor & 1 & 1 & 0 & 0 & 0 & 0 & 1 & 0 & \multirow{3}{*}{0.333} \\
\hline Brenner borderline tumor & 1 & 1 & 0 & 0 & 0 & 0 & 1 & 0 & \\
\hline Transitional cell carcinoma & 0 & - & - & - & - & - & - & - & \\
\hline Endometrioid tumors & 6 & & & & & & & & \\
\hline Benign(cystadenoma) & 5 & 3 & 2 & 0 & 0 & 4 & 1 & 0 & \multirow{3}{*}{0.061} \\
\hline $\begin{array}{l}\text { Endometrioid borderline } \\
\text { tumor }\end{array}$ & 0 & - & - & - & - & - & - & - & \\
\hline \multirow[t]{2}{*}{ Endometrioid adenocarcinoma } & 1 & 1 & 0 & 0 & 0 & 1 & 0 & 0 & \\
\hline & 66 & 58 & 4 & 4 & 5 & 10 & 16 & 35 & $<0.001$ \\
\hline
\end{tabular}

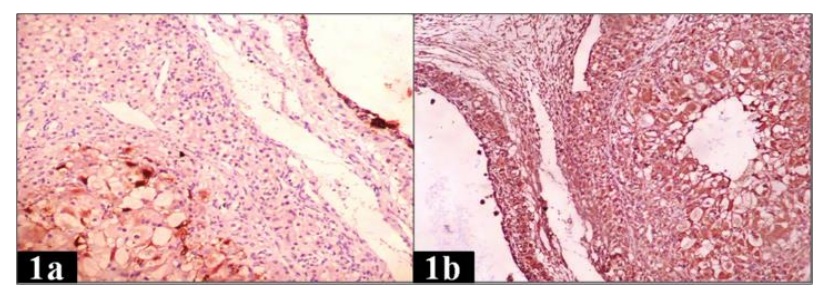

Fig 1. IHC expression of CK AE1/AE3 and vimentin in normal ovary

(a) Surface epithelium showing $3+$ and granulosa cells showing 2+ CK immunostaining (100X)

(b) Surface epithelium showing 1+ and granulosa cells showing 3+ vimentin immunostaining (400X)

In the normal ovary, pancytokeratin was positive with high intensity on the surface epithelium, with mildly appreciable vimentin reactivity. Vimentin $3+$ was observed in endothelium, granulosa cells of ovarian follicle and ovarian cortical stroma.
Granulosa cells showed moderate coexpression of cytokeratin with vimentin (Figure 1).

All ovarian epithelial tumors were positive for cytokeratin expression. Serous tumors of ovary exhibited vimentin expression depending on the degree of differentiation. Vimentin expression was absent in $24 / 28(85.71 \%)$ of cases withbenign serous tumors. All borderline and malignant serous tumors were positive for vimentin with different degrees of positivity. The $3+$ grade for vimentin was present in 5/8 (62.5\%) and 2+ grade in 2/8 (28.57\%) of cases withserous adenocarcinoma. No borderline serous tumor showed grade $3+$ expression for vimentin, $3 / 5(60 \%)$ of cases were $2+$ grade and $2 / 5(40 \%)$ were $1+$ grade. The relationship between $\mathrm{CK}$ and vimentin expression were statistically significant (figures 2, 3, and 4). 


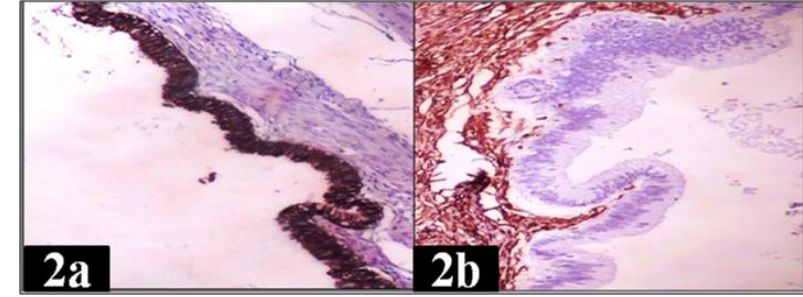

Fig 2. IHC expression of CK AE1/AE3 and vimentin in serous cystadenoma ovary

(a) Strong membranous staining of CK (100X)

(b) No immunostaining of vimentin (100X)

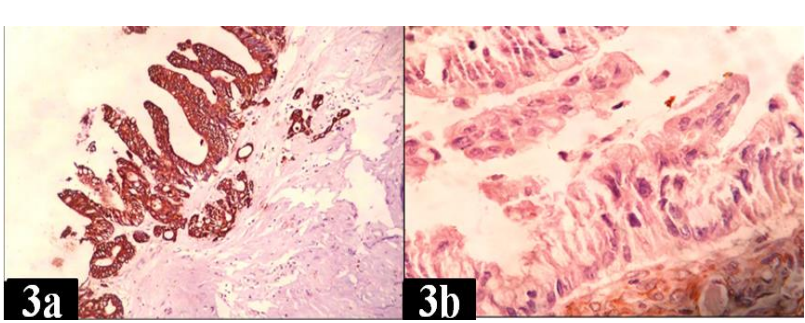

Fig 3. IHC expression of CK AE1/AE3 and vimentin in borderline serous tumor of ovary

(a) Strong membranous staining of $\mathrm{CK}(100 \mathrm{X})$

(b) Mild cytoplasmic expression of vimentin (400X)

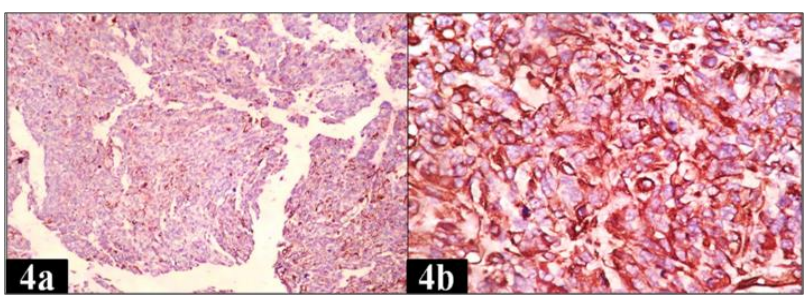

Fig 4. IHC expression of CK AE1/AE3 and vimentin in malignant serous tumor of ovary

(a) Membranous staining of CK in few tumor cells (100X)

(b) Strong cytoplasmic expression of vimentin (400X)

Mucinous tumors of ovary exhibited much less vimentin expression unrelated to the degree of differentiation. Out of seven benign mucinous tumors, six $(85.71 \%)$ were negative for vimentin and one $(14.29 \%)$ case exhibited mild vimentin positivity. Neither borderline mucinous tumors nor the mucinous adenocarcinoma displayed high grade vimentin positivity. Mild vimentin expression was noticed in $4 / 6(66.67 \%)$ cases of borderline mucinous tumors and $1 / 4$ (25\%) cases in mucinous adenocarcinoma. The rest of the borderline and malignant mucinous tumors were negative for vimentin. CK and vimentin expression were significantly associated with mucinous tumors of ovary (figures5, 6, and 7).



Fig 5. IHC expression of CK AE1/AE3 and vimentin in benign mucinous tumor of ovary

(a) Strong membranous staining of CK (400X)

(b) No immunostaining of vimentin (400X)

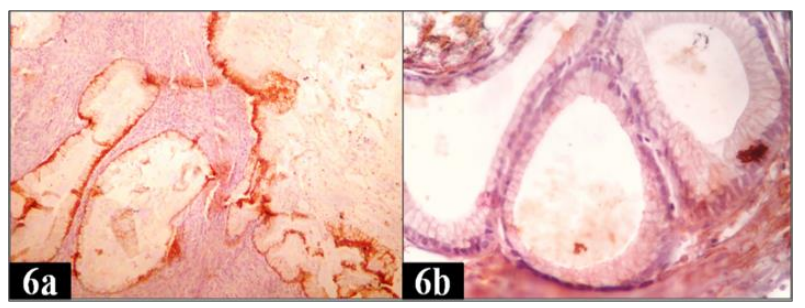

Fig 6. IHC expression of CK AE1/AE3 and vimentin in borderline mucinous tumor of ovary

(a) Strong membranous staining of CK (100X)

(b) Mild cytoplasmic expression of vimentin (400X)

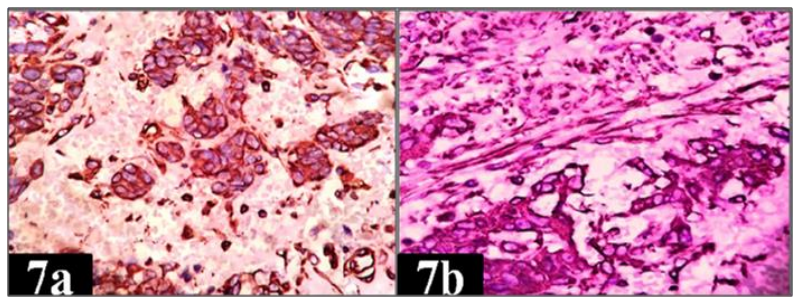

Fig 7. IHC expression of CK AE1/AE3 and vimentin in malignant mucinous tumor of ovary

(a)Strong membranous staining of CK (400X)

(b) No immunostaining of vimentin (400X)

Endometrioid and transitional tumors illustrated vimentin co-positivity, but much less related to the degree of differentiation. Both benign (4/5 cases; $80 \%)$ and malignant $(1 / 1 ; 100 \%)$ endometrioid tumors showed $2+$ grade for vimentin. Both benign and borderline transitional tumors showed $1+$ grade for vimentin. However, the relationship was not statistically significant.

\section{Discussion}

The analysis of distinct classes of intermediate filament (IF) protein expressed by immunehistochemistry (IHC) in neoplastic cells, is acknowledged as a valuable diagnostic tool. The current study precisely examined all types of pure ovarian epithelial tumors to ascertain the profiles of 
cytokeratin and vimentin and to address characteristically the frequency and pattern of intermediate filaments coexpression. Such information may be diagnostically useful since it is shown that coexpression may occur in a number of malignant neoplasms such as renal cell, endometrial, papillary thyroid carcinomas, some ovarian carcinomas, etc (5).

In normal ovaries, surface epithelial cells are strongly immunostained for CK AE1/AE3 and faintly expressed vimentin. The expression of $\mathrm{CK}$ and vimentin in ovarian surface epithelium was not surprising depending on its embryonic origin from the coelomic epithelium, which demonstrates similar expression (6). Granulosa cells of ovarian follicles showed the presence of both CK AE1/AE3 and vimentin in all stages of development, with some deviations, depending on the developmental stage. Granulosa cells of primary and secondary follicles, moderately represented CK AE1/AE3 expression with the decrease in number of positive cells in the Graafian follicles. On the contrary, immunostaining of vimentin in granulosa cells usually remained unchanged during all the follicular development. The expression of CKs and vimentin in ovarian granulosa cells are also described in the literature written in English (6-8). These findings are backed by the origin of granulosa cells from mesonephricduct epithelium, where this expression was observed (9).

CK and vimentin expression was present in $31 / 66$ (46.97\%) of cases with epithelial tumors of ovary. It was associated with the type of tumor as well as the degree of differentiation.

In serous tumors, vimentin expression was inversely related to the degree of differentiation. The predominant pattern of staining was peripheral (membranous). Degree of positivity for vimentin was less in benign serous tumors (negative in $85.71 \%$ cases), compared with borderline and malignant tumors. Thus, the absence of vimentin expression was highly suggestive, but not diagnostic for benign differentiation of serous tumor of ovary. Also, it wa

$\mathrm{s}$ found that no borderline serous tumors showed $3+$ for vimentin, but $2+(60 \%)$ and $1+(40 \%)$ were noticed and $62.50 \%$ of malignant serous tumors demonstrated $3+$ staining depth. Therefore, high intensity of staining for vimentin was indicative for malignant differen-tiation in serous tumors of ovary. It was indicated that vimentin expression in epithelial cells may be explained as epithelial to mesenchymal transition and reduced cell-to-cell contact (10). This also suggested that increased expression of vimentin in malignant epithelial ovarian tumors was certainly related to the dedifferentiation of epithelial cells. This view was further supported by the well-established fact that vimentin belonged to the early response competence gene family and different cells during their differentiation replaced vimentin partially or entirely with their cell specific intermediate filament subunit. Moreover, recent experiments linked over expression of vimentin with inhibition of differentiation (11).

A study conducted by Viale et al., showed that in serous tumors, the expression of vimentin was related to the degree of tumor differentiation (12). But, it was consistently identifiable in the better differentiated tumors; against the current study where it was defined in borderline and malignant serous tumors. In a study conducted by Dabbs DJ et al., $42 \%$ serous carcinoma displayed vimentin expression, but was not related to the degree of differentiation (13). Tenghou et al., demonstrated strong vimentin and cytokeratin expression in all six cases of serous carcinoma correlated with the current study where all cases were positive for vimentin with $5 / 8(62.5 \%)$ cases showing strong positivity (14).

In a study conducted by Matsuzaki et al., 5/23 (21.73\%) cases with serous cystadenoma showed vimentin expression. It was similar to the current study showing vimentin expression in 4/28 (14.29\%) cases of serous cystadenoma (15).

In ovarian mucinous tumors, the pattern of epithelial cells staining with vimentin was peripheral. The intermediate filaments coexpression was associated with the type of tumor and unrelated to the degree of differentiation. It was similar to the researchers conducted by Viale et al., and Dabbs DJ et al. $(12,13)$ Hence, no case of strong vimentin positivity was identified in benign, borderline, and malignant mucinous tumors, which was correlated with the current study. 
Dabbs DJ et al., conducted a study on 79 cases of epithelial tumors of ovary and analyzed eight cases of mucinous cystadenocarcinoma. No case of mucinous adenocarcinoma with vimentin expression was detected by them (13). In a recent publication by Kir $\mathrm{G}$ et al., vimentin was present in $18 \%$ of mucinous ovarian carcinomas. [16] According to the current study, 3/4 (75\%) of the cases were negative for vimentin and only one (25\%) case of mucinous adenocarcinoma displayed mild vimentin positivity, similar to the study by Kir $\mathrm{G}$ et al.

In a study by Matsuzaki et al., no vimentin expression was observed in the studied 20 mucinous cystadenoma cases (14). According to the current study, only $1 / 7$ (14.28\%) mucinous cystadenoma cases displayed mild vimentin positivity.

Thus, it combined a low sensitivity with high specificity to identify the type of tumor as serous; endometrioid and transitional tumors displayed stronger vimentin expression than mucinous tumors. Absence of vimentin decoration in epithelial tumors of ovary is highly suggestive of mucinous tumors unrelated to the degree of differentiation. The scant expression of vimentin in mucinous tumors may be correlated to the predominance of 'colonic type' mucinous cells.

In the ovarian endometrioid carcinomas, no association of tumor differentiation was found with the degree of expression of intermediate filaments. Both benign endometrioid $(4 / 5 ; 80 \%)$ and malignant endometrioid tumors $(1 / 1 ; 100 \%)$ showed perinuclear staining with moderate vimentin expression. In a study by Viale et al., the number of endometrioid carcinomas demonstrating perinuclear vimentin positivity were $71 \%$. However, correlation with the previous studies was difficult, since different antibody and markedly different digestion procedures were used in the studies.

The ovarian surface epithelium along with endometrium, endocervix, and the upper part of the vagina are a form of mullerian epithelium. Their intermediate filaments composition is identical to mesothelium. Of the mullerian epithelial tissue, endometrium displays vimentin normally, as well as in neoplasms. In the normal proliferative endometrium expression of vimentin,it exhibits cyclic sensitivity to the hormonal milieu. The greater regularity with which vimentin is expressed in endometrial and ovarian endometrioid carcinomas in comparison to serous ovarian carcinomas may reflect differential hormonal sensitivity of the neoplastic cells. This phenomenon also correlates with normal endometrial differentiation in which the proliferating endometrium (the architecture of which is changing daily) routinely expresses vimentin whereas in the differentiated secretary endometrium, vimentin is largely absent. Thus, both benign and malignant endometrioid tumors of ovary express vimentin intermediate filament regardless of the degree of differentiation (17).

Transitional cell tumors of the ovary are a relatively unusual neoplasm. They constitute only $1.4 \%$ to $2.5 \%$ of epithelial tumors of ovary and have a predilection for the postmenopausal females. Most of them are benign and less than 5\% are proliferating or borderline (18). Only two cases $(0.03 \%)$ of transitional cell tumors were noticed, one benign and one borderline and both showed mild vimentin decoration. Thus, intermediate filaments co-expression is not linked to the degree of differentiation in Brenner tumors of ovary. Previously, no studies were conducted on the cytokeratin and vimentin expression in ovarian transitional cell tumors.

\section{Conclusion}

Immunohistochemically, there are distinct differences of intermediate filament profile expression within the groups of benign, borderline, and malignant common epithelial ovarian neoplasms. Epithelial-mesenchymal transition such as processes might be involved in the pathogenesis of serous tumors of ovary. Cytokeratin-vimentin expression occurs in all ovarian serous carcinoma and endometrioid adenocarcinoma. Thus, these tumors can be added to the list of neoplasms, which coexpress cytokeratin and vimentin.

\section{Conflict of interest}

There was no conflict of interest.

\section{References}

1. Rein BJD, Gupta S, Dada R, Safi J, Michener C, Agarwal A. Potential Markers for 
Detection and Monitoring of Ovarian Cancer. Journal of Oncology. 2011;2011

2. Riccardi E, Grieco V, Verganti S, Finazzi M. Immunohistochemical diagnosis of canine ovarian epithelial and granulosa cell tumors. J Vet Diagn Invest.2007;19(4):431-5

3. Herrmann H, Aebi U. Intermediate filaments and their associates: multi-talented structural elements specifying cytoarchitecture and cytodynamics. Curr Opin Cell Biol. 2000;12(1):7990 .

4. Kaku T, Ogawa S, Kawano Y, Ohishi Y, Kobayashi H, Hirakawa $\mathrm{T}$, et al. Histological classification of ovarian cancer.Med Electron Microsc.2003;36(1):9-17.

5. Bahrami A, Truong LD, Ro JY. Undifferentiated tumor: true identity by immunohistochemistry.Arch Pathol Lab Med.2008;132:326-48.

6. Loffler S, Horn LC, Weber W, SpanelBorowski K: 2000, The transient disappearance of cytokeratin in human fetal and adult ovaries. Anat Embryol (Berl).2000;201:207-15

7. Benjamin E, Law S, Borrow LG. Intermediate filaments cytokeratin and vimentin in ovarian sex cord-stromal tumors with correlative studies in adult and fetal ovaries. J Pathol. 1987; 152(4):253-63.

8. Czernobilsky B, Moll R, Levy R, Franke WW. Coexpression of cytokeratin and vimentin filaments in mesothelial, granulosa and rete ovarii cells of the human ovary. Eur J Cell Biol.1985; 37:175-90.

9. Holthöfer H, Miettinen A, Lehto VP, Lehtonen E, Virtanen I. Expression of vimentin and cytokeratin types of intermediate filament proteins in developing and adult human kidneys. Lab Invest. 1984;50(5):552-9.

10. Liu C-Y, Lin H-H, Tang M-J, Wang YK. Vimentin contributes to epithelial-mesenchymal transition cancer cell mechanics by mediating

\section{How to Cite This Article:}

cytoskeletal organization and focal adhesion maturation. Oncotarget. 2015;6(18):15966-83.

11. Nakopoulou L, Stefanaki k, Janinis J, Mastrominas M. Immunohistochemical Expression of placental alkaline phosphatase and vimentin in Epithelial Ovarian Neoplasms. Acta Oncologica. 1995;34(4): 511-5.

12. Viale G, Gambacorta M, Dell'Orto P, Coggi G.Coexpression of cytokeratins and vimentin in common epithelial tumours of the ovary: an immunocytochemical study of eightythree cases. Virchows Arch A Pathol Anat Histopathol. 1988;413(2):91-101.

13. Dabbs D. J. and Geisinger K R.Common epithelial ovarian tumors. Immunohistochemical intermediate filament profiles. Cancer.1988; 62: 368-74.

14. Hou T, Liang D, He J, Chen X, Zhang Y. Primary peritoneal serous carcinoma: a clinicopathological and immunohistochemical study of six cases. International Journal of Clinical and Experimental Pathology. 2012;5(8):762-9.

15. Matsuzaki S, Darcha C. Epithelial to mesenchymal transition- like and mesenchymal to epithelial transition- like processes might be involved in the pathogenesis of pelvic endometriosis. Hum Reprod.2012;27:712-21 .

16. Kir G, Gurbuz A, Karateke A, Kir M. Clinicopathologic and immunohistochemical profile of ovarian metastases from colorectal carcinoma. World Journal of Gastrointestinal Surgery. 2010;2(4):109-16.

17. Dabbs DJ, Geisinger KR, Noms HT. Intermediate filaments in endometrial and endocervical carcinomas: The diagnostic utility of vimentin patterns. Am J Surg Pathol 1986; 10368576

18. Borah T, Mahanta RK, Bora BD, Saikia S. Brenner tumor of ovary: An incidental finding. Journal of Mid-Life Health. 2011;2(1):40-1.

Goel A, Rao, Santhi V, Byna S, Grandhi B, Conjeevaram J. Immunohistochemical Characterization of Normal Ovary and Common Epithelial Ovarian Neoplasm with a Monoclonal Antibody to Cytokeratin and Vimentin. Iran J Pathol, 2018; 13(1): 23-29. 